\title{
Enterovesical fistula and acute pyelonephritis in renal transplantation. Role of ultrasound
}

\author{
Antonio De Pascalis, Alessandro D'Amelio \\ Nephrology and Dialysis Unit, V Fazzi Hospital, Lecce, Italy.
}

\begin{abstract}
Summary The enterovesical fistula is a communication between the urinary tract and the colon and is a rare complication of various inflammatory and cancer diseases. The most frequent cause is represented by diverticulitis of the sigmoid colon and less frequently from Crohn's disease, tumors of the colon and bladder, trauma, radiation therapy and appendicitis. In this report we describe the occurrence of an enterovesical fistula in a patient with renal allograft from a cadaveric donor, which onsetted with signs of acute pyelonephritis and pneumaturia due to diverticulitis of the sigmoid colon, clinically silent. The ultrasound in the diagnosis of enterovesical fistula, yet with a minor role compared to computed tomography (CT), is fundamental being always the first level examination.
\end{abstract}

KEY WORDS: Fistula; Bladder; Pyelonephritis.

Submitted 3 October 2014; Accepted 31 October 2014

\section{Case report}

A 64 years old male, with a kidney transplantation from cadaveric donor for about 5 years and treated with prednisone, tacrolimus and mycophenolate, presented to the emergency room with signs and symptoms suggestive of urinary sepsis (fever, hypotension, dysuria, pain in the right iliac fossa, where the graft was allocated).

Laboratory tests showed: mild worsening of renal function (creatinine $2.4 \mathrm{mg} / \mathrm{dL}$ ), neutrophilic leukocytosis (GB 14.440/ml with 91\% neutrophils), elevated inflammatory markers (ESR 120, CRP $153 \mathrm{mg} / \mathrm{dL}$, procalcitonin $5 \mathrm{mg} / \mathrm{L}$ ), urine examination revealed leukocyturia, hematuria, bacteriuria.

The patient reported that he had noticed at home the issue of foamy urine. An ultrasound of the kidney and urinary tract showed a transplanted kidney of globular shape with multiple hypoechoic areas suggestive for hydroureteronephrosis grade II, presence of intrapyelic hyperechogenic material and a diffusely thickened bladder (Figure 1); color Doppler evaluation showed an increased intraparenchymal Doppler RI (0.84). We therefore decided to perform a computed tomography (CT) scan which confirmed the signs of acute pyelonephritis of the graft, showing also the presence of hydro-air level and the presence of a fistula between the sigmoid colon and the left side wall of the bladder, associated with numerous diverticula of the colon (Figure 2). After starting antibiotic therapy with ciprofloxacin and teicoplanin we assisted to a dramatic improvement of the clinical picture. Subsequentely patient underwent surgical sigmoidectomy and bladder repair.

\section{Discussion}

Enterovesical fistulas account for over $80 \%$ of fistulas between digestive and urinary tract and, the most frequent between the bladder and sigmoid colon. Diverticulitis of the colon is the most frequent cause of fistula with the bladder, followed by Crohn's disease and colon cancers (1). In the case reported the patient had complicated diverticulitis of the colon, with a very few symptoms (the patient reported only alternating diarrhea and constipation) evidently because of steroid therapy. However, the clinical picture dominating the majority of patients with enterovesical fistula, as reported in the literature and as moreover observed in our case, are the urinary symptoms (fever, dysuria, or even pneumaturia and fecaluria) (2).

The occurrence of pneumaturia is a highly specific sign of a communication between the intestine and bladder, reported in $60-85 \%$ of cases described in the literature (3). In the literature there are few reports about the ultrasound scan for pneumaturia and then about the diagnosis of enterovescical fistula by ultrasound examination. The typical signs described are: the presence of the socalled echogenic beak, i.e. an area of hyperechoic beak, between the bladder and adjacent bowel, without solution of continuity; the passage of air and echogenic material in the urine, after the abdominal compression; the presence of irregular hyperechoic foci with shadow cone back (4).

The most sensitive and specific test is, of course, CT which allows you to directly highlight the presence of the fistula, the cause that generated it and its associated complications (5). Less sensitive and specific and therefore not indicated, is the intravenous urography.

Cystoscopy is obviously highly sensitive and specific and is often performed as an examination of the level before the $\mathrm{CT}$ in suspected enteric fistula with bladder.

No conflict of interest declared. 
Figure 1.

US. Hyperechoic material in pelvis with acoustic shadowing and thickened ureteral wall.

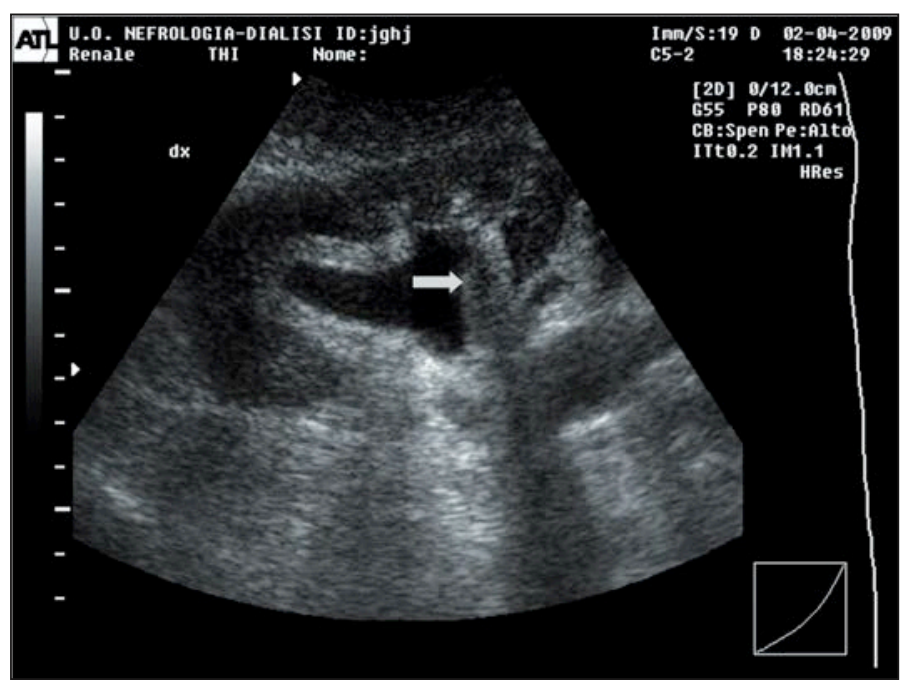

Figure 2.

CT. Air-urine level in the bladder.

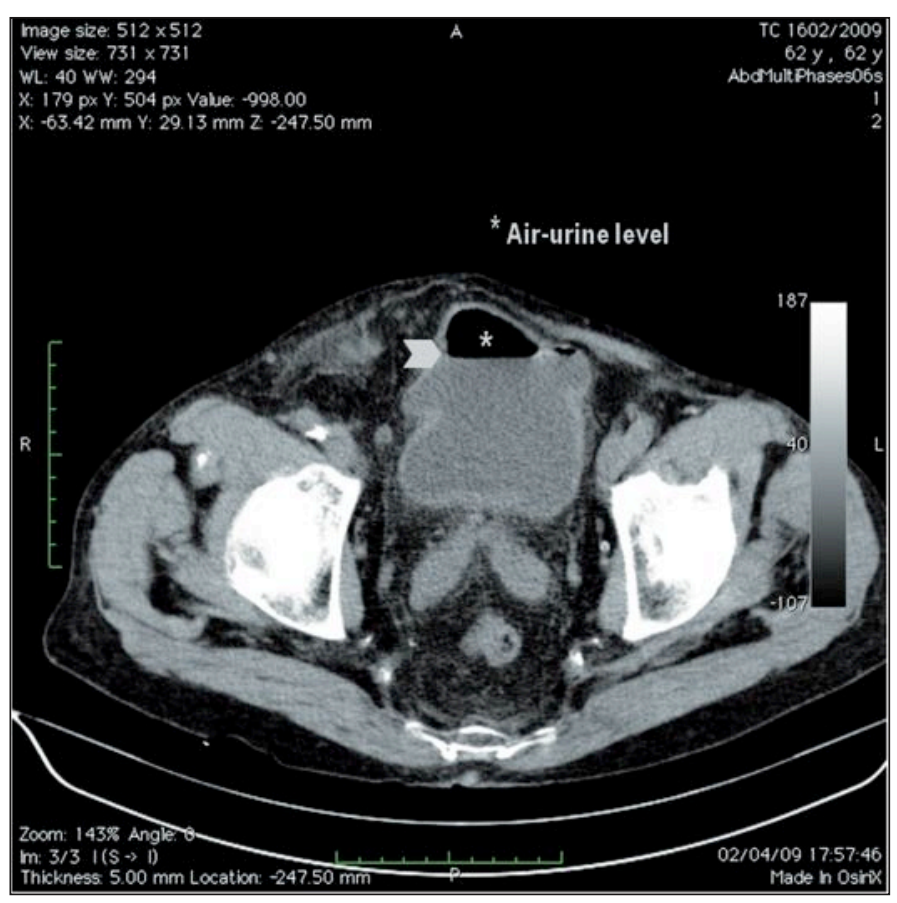

In conclusion, we reported a case, one of the few in the literature, of enterovesical fistula secondary to diverticulitis of the colon in a kidney transplanted patient. Concomitant immunosuppressive therapy and steroids in particular, has probably masked the clinical picture until the onset of acute graft pyelonephritis.

The ultrasound in the diagnosis of enterovesical fistula, yet with a minor role compared to CT, is fundamental being always the first level examination because of its non-invasiveness, repeatability and low cost, and it can provide guidelines that address the diagnosis.

\section{BiBLIOGRAPHY}

1. Krco MJ, Jacobs SC, Malangoni MA, Lawson RK. Colovescical fistulas. Urology. 1984; 23:340-342.

2. VesaLlane's J, Llado Carbonell C, Valverde Sintas J, Bielsa Gali O. Fistulas vesico-sigmoideas. Arch EspUrol. 1991; 44:1133-1138.

3. Kirsh GM, Hampel N, Shuck JM, Resnick MI. Diagnosis and management of vesicoenteric fistulas. Surg Gynecol Obstet. 1991; 173:91-97.

4. Long MA, Boultbee JE. Case report: the transabdominal ultrasound appearances of a colovesical fistula. $\mathrm{Br} \mathrm{J}$ Radiol. 1993; 66:465-467.

5. Jarrett TW, Vaughan ED. Accuracy of computerized tomography in the diagnosis of colovesical fistula secondary to diverticular disease. J Urol. 1995; 153:44-46.

\section{Correspondence}

Antonio De Pascalis, MD (Corresponding Author) depascalis.a@libero.it

Alessandro D'Amelio, MD

Nephrology and Dialysis Unit, V Fazzi Hospital

Piazza Muratore 1 - 73100 Lecce, Italy 